\title{
Photons and jets at the Tevatron.
}

\author{
Dmitry Bandurin \\ Fermilab, PO Box 500, Batavia, IL, 60510
}

\begin{abstract}
Presented are the Run II QCD preliminary results of D0 and CDF Collaborations on the measurements of inclusive jet cross sections as well as recently published results on the inclusive and di-photon cross sections.
\end{abstract}

Keywords: prompt photons, hadron jets

PACS: $13.85 . \mathrm{Qk}, 12.38 . \mathrm{Qk}$

\section{INTRODUCTION.}

Hadron colliders provide us lots of data for studying QCD dynamics, its parameters and particle structures. These studies are very interesting and useful as for the physics itself as for expected new physics processes. The later at hadron colliders are most likely to go via QCD subprocesses and, at the same time, the dominating background to them is usually caused by QCD subprocesses as well. Thus, searches of most new physics both at Tevatron Run II and LHC will not be possible without our solid knowledge of many aspects of QCD.

It is well known that precision QCD calculations and their experimental tests at hadron colliders are difficult. At theoretical side the difficulty is mainly caused by our limited knowledge of parton fragmentation mechanism, diffractive processes, underlying events and particle structure functions. While the experiments are limited by finite detector or accelerator capabilities. QCD is extremely diverse it terms of final products and deals with production of jets, photons, $\mathrm{W}$ and $\mathrm{Z}$ bosons, and heavy flavor quarks.

\section{JET AND PHOTON MEASUREMENTS.}

\section{Inclusive jet cross section.}

One of the tools for the QCD studies is measurement of inclusive jet cross section. At large transverse momenta $\left(p_{T}\right)$ this cross section is directly sensitive to $\alpha_{s}$ and parton distribution functions (PDFs). As we know, inclusion of Tevatron Run I cross sections substantially modified PDF behavior, especially gluon PDF at high $x$. But the gluon PDF uncertainty is still big and grows in the region of high $Q^{2}$. In spite of just $10 \%$ difference of $\sqrt{s}$ between Run I and Run II (1.8 TeV vs. $2 \mathrm{TeV})$, inclusive jet cross sections substantially differ at high $p_{T}$ 's, e.g. by about $300 \%$ at $p_{T}=500 \mathrm{GeV}$. Over a wide $p_{T}$ range the $q g$ scattering dominates in the jet production $(80 \%$ at $40 \mathrm{GeV}$ and $50 \%$ at $300 \mathrm{GeV}$ ) and even at $p_{T}=500 \mathrm{GeV}$ its contribution is around $30 \%$. It means that in this domain the jet cross section is still sensitive to gluon PDF and can 

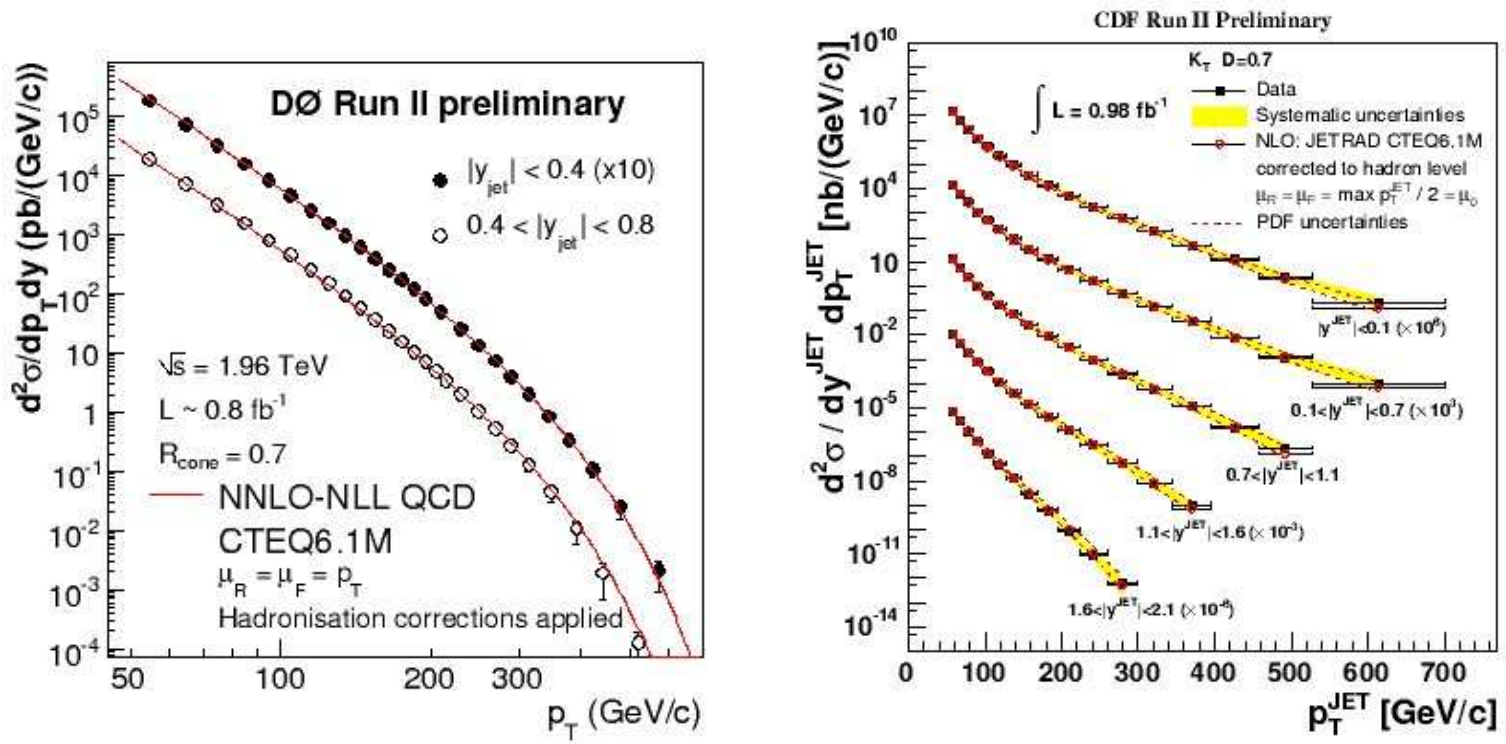

FIGURE 1. D0 (left) and CDF (right) inclusive jet cross sections.

provide points for $Q^{2} \approx 10^{5} \mathrm{GeV}$ and $x \approx 0.5-0.6$. D0 experiment presents a new preliminary measurement of the inclusive jet cross section in ${ }^{-} p p$ collisions based on a integrated luminosity of about $0.8 \mathrm{fb}^{-1}$. Jets are reconstructed using an iterative cone Midpoint algorithm (free of infrared singularities) with cone radius $R_{\text {cone }}=0.7$. The inclusive jet cross section is presented in Fig.1 (left) as a function of $p_{T}$ in two rapidity intervals. It covers the range up to almost $600 \mathrm{GeV}$. Predictions from perturbative QCD in next-to-leading order (NLO), plus threshold corrections in the 2-loop accuracy describe the shape in $p_{T}$ within uncertainties. Uncertainties on jet inclusive $p_{T}$ cross section are dominated by jet energy scale (JES) statistical uncertainties and should be reduced by 2007 year. It is worth emphasizing that even current cross section systematic uncertainties are comparable with PDF ones and allow to make limitation on the PDF behaviour, especially at high $p_{T}$ 's.

Analogous measurement of differential jet cross section with improved cone $R=0.7$ algorithm was done by CDF in the central rapidity region of $0.1<|y|<0.7$ with $p_{T}$ up to $620 \mathrm{GeV}$. Data are compared to QCD NLO (Ellis-Kunzt-Sopper) predictions. Data corrected to parton level (while in previous Run I measurement the hadronization corrections were not applied.)

Another jet inclusive $p_{T}$ cross section using Ellis-Sopper $k_{T}$ algorithm was measured in CDF (Fig.1, right plot). The measurement was done in five jet rapidity bins up to $|y|<2.1$ to cover separately different parton $x$ ranges. NLO QCD calculations with JETRAD are corrected for hadronization and underlying events. Results are based at almost $1 \mathrm{fb}^{-1}$ of data and fully unfolded to hadron level. Data uncertainty is comparable or even smaller than PDF uncertainty at high $y$ regions and should allow to further constrain PDF, especially using high rapidity bins. 

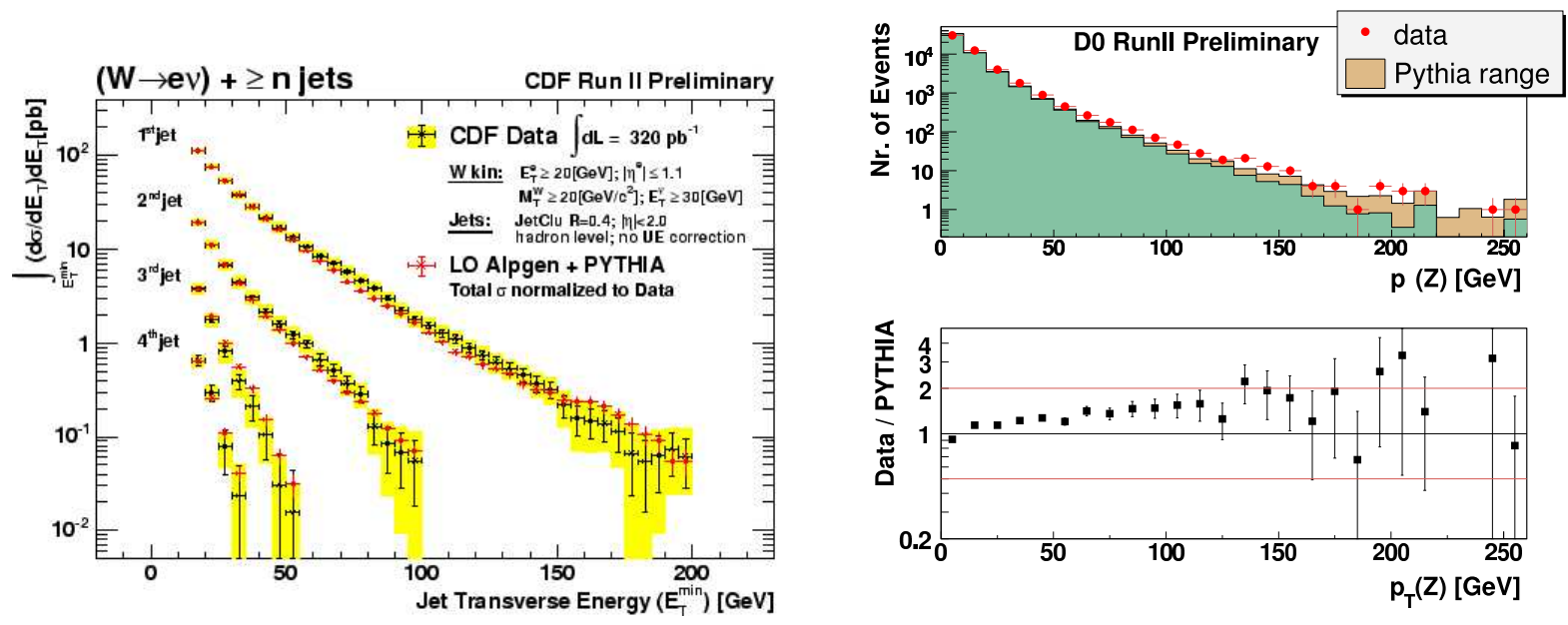

FIGURE 2. W+jets (left) and Z+jets (right) events.

\section{W/Z+jet measurements.}

Associated production of vector bosons with jets is very good tool for testing QCD at hadron colliders. In addition, 'W/Z + jet' production is an important background to WH and $\mathrm{ZH}$ production. Fig.2 (left) presents CDF results on measurement of 'W+jets' differential cross sections that was done as a function of jet $p_{T}, \eta-\phi$ distance $(R)$ between jets, and jet-jet invariant mass. It was compared with LO theory (ALPGEN+PYTHIA, normalized to the total data cross section). In general, one can state that data points within uncertainties agree with theoretical predictions, but there are tendencies to differ at leading jet high $p_{T}$, in the shapes of invariant mass and di-jet $R$ distributions (not shown here).

Analogous study, this time with 'Z+jets' events, was done in D0 in comparison with MC generators (PYTHIA and SHERPA) predictions (Fig.2, right plot). Data are in a good agreement with MC predictions, but central prediction from PYTHIA contains fewer hard jets than seen in data and discrepancy increases with jet multiplicity. One can also note that $\Delta \phi(j e t, j e t)$ distribution shows significant peak at $\pi$ in PYTHIA sample but not in data (not shown here).

\section{Inclusive photon cross section.}

Main motivation for the photon measurements is that direct photon emerge unaltered from the hard scattering and due to electromagnetic interaction with quarks provide direct probe of the hard scattering dynamics. In addition, photons are free from complications caused by jet fragmentation and systematics caused by their identifications and measurements. Photons may provide direct information on gluon PDF : gluon involved at LO in contrast to DIS or DY processes. They are also good source for testing gluon resummation techniques and various models of gluon radiation. As in case of jets, it is worth mentioning that understanding of photon production is prerequisite in our 

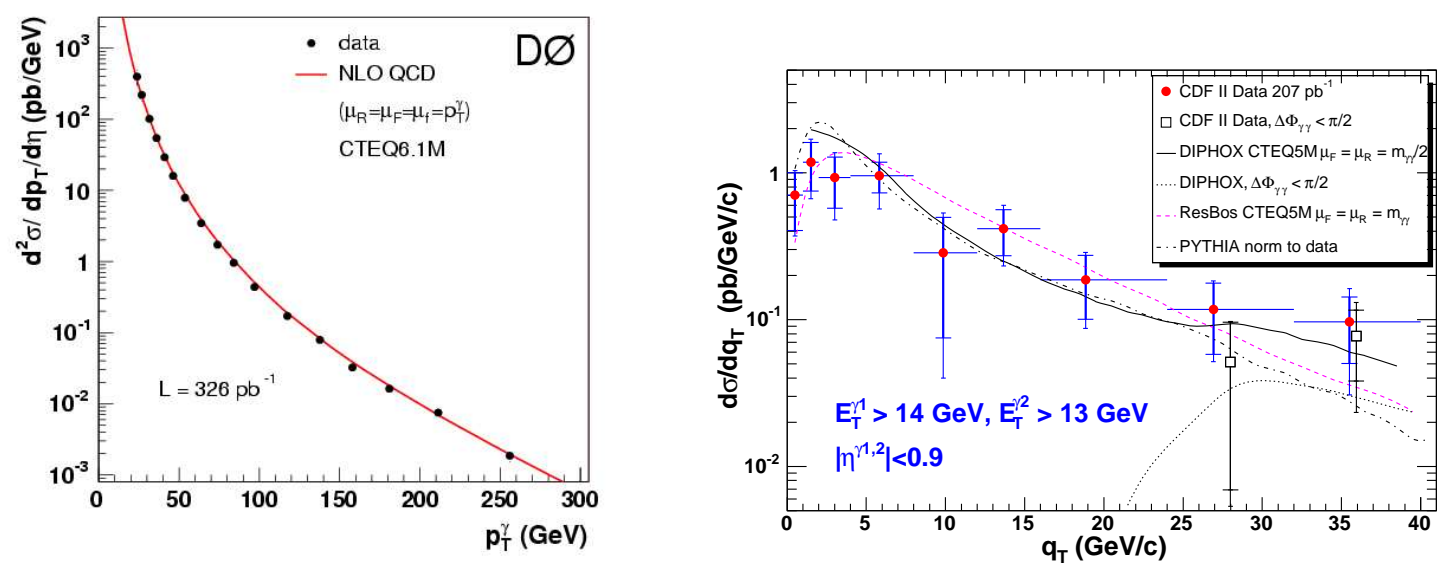

FIGURE 3. D0 inclusive jet cross section.

searches of new physics.

Inclusive photon cross section was measured in D0 in the central rapidity region of $|y|<0.9$ and in the widest $p_{T}^{\gamma}$ range that has ever been covered before: $23<p_{T}^{\gamma}<300$ $\mathrm{GeV}$ (left plot of Fig.3). Data are in agreement with NLO pQCD predictions done with JETPHOX package and by W. Vogelsang [1].

\section{Di-photons.}

CDF Collaboration has measured di-photon cross sections over di-photon invariant mass spectrum, azimuthal angle and total transverse momentum of the pair $q_{T}$ (right plot of Fig.3). Data were compared with theoretical predictions done with DIPHOX and ResBos packages [2]. Both programs agree with data within uncertainties, but DIPHOX describes better region of high $q_{T}$, small $\Delta \phi$ and low mass due to account of NLO fragmentation contribution. While ResBos takes into account resummed gluon contribution and describes better low $q_{T}$ and high $\Delta \phi$.

\section{ACKNOWLEDGMENTS}

I am extremely grateful to D0 and CDF QCD groups as well as to E.L. Berger for very fruitful discussions.

\section{REFERENCES}

1. D0 Collaboration, V. Abazov at al, hep-ex/0511054, To be published in Phys.Lett.B.

2. CDF Collaboration, D. Acosta at al, hep-ex/0412050, Phys.Rev.Lett.95 022003 (2005). 